\title{
Rickettsioses as Major Etiologies of Unrecognized Acute Febrile Illness, Sabah, East Malaysia
}

Matthew J. Grigg, Timothy William, Emily G. Clemens, Kaajal Patel, Arjun Chandna, Christopher S. Wilkes, Bridget E. Barber, Nicholas M. Anstey, J. Stephen Dumler, Tsin W. Yeo, Megan E. Reller

\section{$\underset{\text { MDUCATION }}{\text { Medsce ACTIVITY }}$}

In support of improving patient care, this activity has been planned and implemented by Medscape, LLC and Emerging Infectious Diseases. Medscape, LLC is jointly accredited by the Accreditation Council for Continuing Medical Education (ACCME), the Accreditation Council for Pharmacy Education (ACPE), and the American Nurses Credentialing Center (ANCC), to provide continuing education for the healthcare team.

Medscape, LLC designates this Journal-based CME activity for a maximum of 1.00 AMA PRA Category 1 Credit(s) ${ }^{\mathrm{TM}}$. Physicians should claim only the credit commensurate with the extent of their participation in the activity.

Successful completion of this CME activity, which includes participation in the evaluation component, enables the participant to earn up to $1.0 \mathrm{MOC}$ points in the American Board of Internal Medicine's (ABIM) Maintenance of Certification (MOC) program. Participants will earn MOC points equivalent to the amount of CME credits claimed for the activity. It is the CME activity provider's responsibility to submit participant completion information to ACCME for the purpose of granting ABIM MOC credit.

All other clinicians completing this activity will be issued a certificate of participation. To participate in this journal CME activity: (1) review the learning objectives and author disclosures; (2) study the education content; (3) take the post-test with a $75 \%$ minimum passing score and complete the evaluation at http://www.medscape.org/journal/eid; and (4) view/print certificate. For CME questions, see page 1643.

Release date: June 18, 2020; Expiration date: June 18, 2021

Learning Objectives

Upon completion of this activity, participants will be able to:

- Evaluate the findings of diagnostic testing for rickettsioses among patients with nonmalarial AFI in Sabah, East Malaysia, from 2013 to 2015

- Assess the clinical and laboratory findings among patients with nonmalarial AFI in Sabah, East Malaysia, who were diagnosed with rickettsioses from 2013 to 2015

- Determine the clinical and public health implications of clinical and epidemiological findings among patients with nonmalarial AFI in Sabah, East Malaysia, who were diagnosed with rickettsioses from 2013 to 2015.

\section{CME Editor}

Jude Rutledge, BA, Technical Writer/Editor, Emerging Infectious Diseases. Disclosure: Jude Rutledge has disclosed no relevant financial relationships.

\section{CME Author}

Laurie Barclay, MD, freelance writer and reviewer, Medscape, LLC. Disclosure: Laurie Barclay, MD, has disclosed no relevant financial relationships.

\section{Authors}

Disclosures: Matthew J. Grigg, MBBS, PhD; Timothy William, MBBS, FRCP(Edin); Emily G. Clemens, MA; Kaajal Patel, MBBS; Arjun Chandna, MD; Christopher S. Wilkes, MBBS; Bridget E. Barber, MBBS, PhD; Nicholas M. Anstey, MBBS, PhD; John Stephen Dumler, MD; Tsin Wen Yeo, MBBS, PhD; and Megan E. Reller, MD, PhD, have disclosed no relevant financial relationships.

Author affiliations: Infectious Diseases Society Sabah-Menzies

School of Health Research, Kota Kinabalu, Malaysia (M.J. Grigg,

T. William, K. Patel, A. Chanda, C.S. Wilkes, B.E. Barber,

N.M. Anstey, T.W. Yeo); Menzies School of Health Research-

Charles Darwin University, Darwin, Northern Territory, Australia

(M.J. Grigg, K. Patel, A. Chanda, C.S. Wilkes, B.E. Barber,

N.M. Anstey, T.W. Yeo); Gleneagles Hospital, Kota Kinabalu

(T. William); Clinical Research Centre, Queen Elizabeth Hospital,

Kota Kinabalu (T. William); QIMR Berghofer Medical Research

Institute, Brisbane, Queensland, Australia (B.E. Barber);
Uniformed Services University of the Health Sciences, Bethesda, Maryland, USA (J.S. Dumler); Lee Kong Chian School of Medicine, Nanyang Technological University, Singapore (T.W. Yeo); Communicable Disease Centre, Institute of Infectious Diseases and Epidemiology, Tan Tock Seng Hospital, Singapore (T.W. Yeo); Duke University, Durham, North Carolina, USA (M.E. Reller); Duke Global Health Institute, Durham (M.E. Reller)

DOI: https://doi.org/10.3201/eid2607.191722 
Orientia tsutsugamushi, spotted fever group rickettsioses, and typhus group rickettsioses (TGR) are reemerging causes of acute febrile illness (AFI) in Southeast Asia. To further delineate extent, we enrolled patients $>4$ weeks of age with nonmalarial AFI in Sabah, Malaysia, during 2013-2015. We confirmed rickettsioses (past or acute, IgG titer $>160)$ in 126/354 (36\%) patients. We confirmed acute rickettsioses (paired 4-fold IgG titer rise to $\geq 160)$ in $38 / 145(26 \%)$ patients: 23 O. tsutsugamushi, 9 spotted fever group, 4 TGR, 1 O. tsutsugamushil spotted fever group, and 10 . tsutsugamushi/TGR. PCR results were positive in $11 / 319(3 \%)$ patients. Confirmed rickettsioses were more common in male adults; agricultural/plantation work and recent forest exposure were risk factors. Dizziness and acute hearing loss but not eschars were reported more often with acute rickettsioses. Only 2 patients were treated with doxycycline. Acute rickettsioses are common (>26\%), underrecognized, and untreated etiologies of AFI in East Malaysia; empirical doxycycline treatment should be considered.

$R_{s}^{\mathrm{i} c}$ ickettsioses and related infections, including scrub typhus, caused by Orientia tsutsugamushi; spotted fever group rickettsioses (SFGR), often called tick typhus; and typhus group rickettsioses (TGR), also called murine typhus, are reemerging and neglected causes of acute febrile illness (AFI) in Southeast Asia (1-4). Serologic confirmation of acute rickettsioses, however, requires demonstration of a 4-fold rise in IgG titer by indirect fluorescent antibody (IFA) because acute-phase IgG might represent prior infection and IgM might indicate recent or cross-reactive antibody (1). Furthermore, serologic testing in the early phase of illness is not helpful because antirickettsial antibodies are often not present within the first 2 weeks.

Rickettsioses have been reported in Malaysia historically (2-4). Malaysia state tertiary hospital data for the period 1994-1999 identified antirickettsial IgG or IgM in 6,442 $(10.6 \%)$ tested samples (IgG or IgM titer $>400$ by the indirect immunoperoxidase test), including $4.9 \%$ O. tsutsugamushi, 3.1\% TGR, and 2.6\% SFGR (5). Although rickettsioses are notifiable diseases according to Malaysia Ministry of Health guidelines (6), routine testing is not conducted, paired acute-phase and convalescent-phase serum samples are rarely available, and only 53 infections were reported nationally during 2009-2015 (7). In the East Malaysia state of Sabah, 2 cases were reported over that period despite a seroprevalence of $5.5 \%$ among 11,037 patients tested in previous years at the state tertiary referral hospital (5) and high $(>50 \%)$ seropositivity reported in febrile patients from certain rural areas (8). We prospectively enrolled patients and performed reference standard diagnostic testing to document and describe the epidemiology and clinical features of confirmed rickettsioses among patients with nonmalarial AFI in Sabah, East Malaysia.

\section{Methods}

\section{Study Sites and Referral System}

We conducted the study during December 12, 2013July 15, 2015, in 2 adjacent district referral hospitals, Kudat and Kota Marudu, in northwest Sabah, Malaysia, on the island of Borneo. These districts cover an area of $3,204 \mathrm{~km}^{2}$ and had an estimated population in 2015 of $\approx 169,000$ (9). According to Malaysia Ministry of Health divisional health facility structures, each district has a single central referral hospital and subdistrict health clinics. The climate is tropical, has no defined dry season, and experiences increased rainfall during November-March. The region consists of both coastal and inland areas with elevations up to 1,000 $\mathrm{m}$ and extensive recent human land-use change from intact forest to oil palm plots and plantations (10).

\section{Study Population}

Patients $>4$ weeks of age with a documented temperature of $\geq 38^{\circ} \mathrm{C}$ within 48 hours of admission to the medical or pediatric wards and a negative malarial blood film were eligible. We obtained consent from the patient or a parent or guardian if the patient was $<18$ years of age; we also obtained assent from persons 7-17 years of age.

\section{Study Procedures}

Trained research nurses used standardized case record forms to collect structured epidemiologic, clinical, and laboratory data, including the admitting clinician's findings on examination, provisional diagnosis, and management. At enrollment, venous blood was obtained for routine and research-related testing after consent. Patients were requested to return for routine hospital outpatient follow-up and convalescent-phase serum sampling 14 days after enrollment.

\section{Laboratory Procedures}

Hematologic and biochemical tests, blood cultures, and malaria smears were performed in the hospital laboratory. Anemia was classified according to World Health Organization age- and sex-based criteria (11). Acute kidney injury was defined per KDIGO criteria (12). 
We conducted IFA testing at the Uniformed Services University for the Health Sciences (Bethesda, MD, USA) to confirm rickettsial infections; 2 experts read each slide, as described $(13,14)$. We screened convalescent-phase serum samples using IFA at 1:80 dilution for IgG to O. tsutsugamushi (Karp strain), SFGR (Rickettsia conorii Malish 7 strain), and TGR (R. typhi); for reactive samples, we tested acute- and convalescent-phase paired serum samples together and titrated positives to 2,560. To evaluate the utility of acute-phase IgM for identifying paired IgGconfirmed acute infections, we tested a subset with paired IgG results for IgM in addition to those without convalescent-phase serum. We screened acutephase serum samples for IgM at 1:40 and titrated positives to 1,280 .

We performed multiplex real-time PCR to detect O. tsutsugamushi (56-kDA major outer membrane protein gene), SFGR (consensus ompA sequence), and TGR (R. typhi $17-\mathrm{kDa}$ lipoprotein precursor gene) on EDTA-anticoagulated blood or buffy coat stored at $-70^{\circ} \mathrm{C}$ as described (15). We tested $5 \mu \mathrm{L}$ of DNA extracted from a $200 \mu \mathrm{L}$ sample of EDTA-anticoagulated whole blood or buffy coat; we required 2 out of 2 or 3 replicates to confirm a positive result by PCR.

\section{Case Definitions}

We used stringent criteria to define rickettsial infections (16), as reported previously $(13,14)$. We defined confirmed rickettsioses as any IgG titer $\geq 160$; confirmed past rickettsial infection as stable or declining IgG titer $\geq 160$; confirmed acute rickettsial infection as a $\geq 4$-fold rise in IgG titer by paired IFA to convalescent-phase titer of $\geq 160$, positive PCR result, or both; confirmed probable acute rickettsial infection as a 2 -fold rise in IgG titer with convalescent-phase titer $\geq 160$ or single IgG titer $\geq 160$ without paired serum samples; and possible rickettsioses as any IgG titer of 80 (acute infection if IgG seroconversion). We distinguished SFGR from TGR when we observed a $\geq 2$-fold difference in SFGR versus TGR titer; if titers were equal, we categorized the infection as group indeterminate.

\section{Statistical Analysis}

We analyzed data by using Stata (StataCorp, https://www.stata.com). We performed 2-group comparisons by using the Student $t$-test for continuous variables with normal distributions or the Wilcoxon-Mann-Whitney test for skewed distributions. For categoric variables, we used a $\chi^{2}$ or Fisher exact test. We calculated odds ratios (ORs) and $95 \%$ CIs by using the Mantel-Haenszel method.

\section{Ethics}

We obtained ethics approval for this study. The study was approved by relevant institutional review boards in Malaysia (National Medical Research Ethics Committee), Australia (Menzies School of Health Research), and the United States (John Hopkins University and Duke University).

\section{Results}

Of 557 patients who met eligibility criteria, 426 $(77 \%)$ consented to enrolment and blood collection; $183(43 \%)$ patients returned for convalescent follow-up (median 13 days; interquartile range (IQR) 12-14 days), and 157 (37\%) had acute-phase serum samples, convalescent-phase serum samples, or both collected (Figure 1). Among the 243 patients who did not attend follow-up appointments, 240 had acute-phase serum samples available. A total of 354 patients had acute-phase serum samples, convalescent-phase serum samples, or both available to test for rickettsioses; of these, 145 (41\%) patients had paired serum samples. Acute-phase whole blood or buffy coat was available for 319 patients. We observed no difference in median age or sex between those patients with and without paired serum samples.

\section{Serologic Diagnosis}

Testing for IgG by IFA confirmed rickettsial infections in 126 of 354 (36\%) patients, including 96 (27\%) with scrub typhus (O. tsutsugamushi infection), 26 (7\%) with SFGR, and 25 (7\%) with TGR (Table 1); 6 patients had both $O$. tsutsugamushi and SFGR, 8 patients both O. tsutsugamushi and TGR, 1 patient both SFGR and TGR, and 3 patients $O$. tsutsugamushi, SFGR, and TGR IgG. We found confirmed past infection (stable or declining IgG titers $\geq 160)$ in $17(12 \%)$ of 145 patients. Patients with serologic evidence of acute or past SFGR infections were older (median 60 years [IQR 45-73 years]) than patients with $O$. tsutsugamushi infection (median 38 years [IQR 24-57 years]; $\mathrm{p}=0.003$ ) but not patients with TGR (median 48 years [IQR 38-58 years]; $p=0.25$ ) infections.

Among patients with paired serum samples, we confirmed 38 (26\% [95\% CI 20-34]) as having acute rickettsial infections based on a 4 -fold rise in IgG titer, including 23 (15\%) with O. tsutsugamushi, 9 (6\%) SFGR, 4 (3\%) TGR, and 2 co-infections (1 O. tsutsugamushi/SFGR and 1 O. tsutsugamushi/TGR [1\% combined]). Most (22/38 [58\%]) cases were associated with IgG seroconversion, including 13/25 (52\%) acute $O$. tsutsugamushi infections, 9/10 (90\%) acute SFGR, and 2/5 (40\%) acute TGR. 


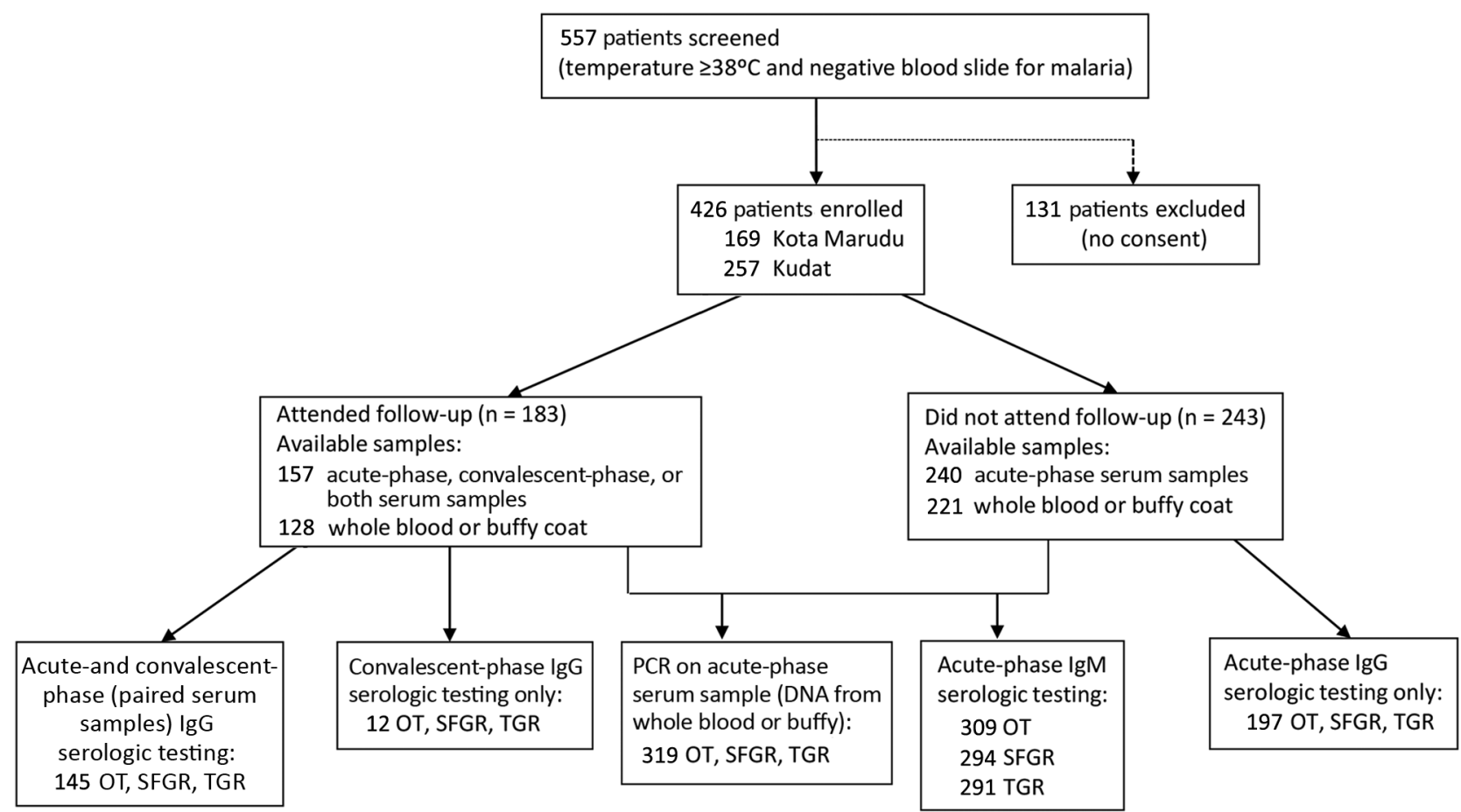

Figure 1. Enrollment flowchart and laboratory testing in a prospective cohort study of acute febrile illness attributable to rickettsioses, Sabah, East Malaysia, 2013-2015. OT, Orientia tsutsugamushi; SFGR, spotted-fever group rickettsiosis; TGR, typhus-group rickettsioses.

Probable acute rickettsial infections were detected in 77/354 (22\%) persons, including 58 with $O$. tsutsugamushi, 12 with SFGR, and 16 with TGR; 70 of these lacked paired serum samples. Among those with paired serum samples, sensitivity of acute-phase IgM for acute rickettsioses was $8 \%(95 \%$ CI 2\%-21\%) and specificity was $94 \%$ (95\% CI 87\%-98\%). We observed possible acute rickettsioses in an additional 37/ 354 patients (10\% [95\% CI 7\%-14\%]) (Appendix Tables 1-3, https://wwwnc.cdc.gov/EID/article/26/7/191722-App1.xlsx).

\section{Diagnosis by PCR}

An additional 11 of 319 patients with acute-phase whole blood or buffy coat available had acute rickettsial infections by PCR. These infections were $1 O$. tsutsugamushi, 8 SFGR, and 2 TGR (Table 1).

\section{Clinical and Laboratory Features of Acute \\ Rickettsial Infections}

Patients with confirmed acute rickettsial infections ( $\mathrm{n}=$ 49; median age 39 years) were equally likely to be male and were of similar age $(\mathrm{p}=0.32)$ to those with probable acute rickettsioses (median age 43 years); both groups were slightly older than those with no rickettsial infection (median age 29 years; $p=0.007$ ) (Table $2)$. The median age of those with acute infections did not differ significantly $(p=0.69)$ : 36 years (IQR $21-51$ years) for $O$. tsutsugamushi, 49 years (IQR 12-58 years) for SFGR, and 38 years (IQR 27-56 years) for TGR. Eight children ( $<15$ years of age) had confirmed acute rickettsial infections (5 SFGR, 2 O. tsutsugamushi, and 1 TGR), of whom $5(63 \%)$ had their infections confirmed by PCR; the youngest was a 6-month-old infant. The median duration of hospitalization for those with confirmed acute rickettsial infections was 5 days (IQR 3-7 days), which was comparable to those without rickettsioses. No deaths were reported.

Clinical and laboratory features of probable acute rickettsial infections were comparable to those with confirmed infections (Table 2), except for less frequent dyspnea ( $17 \%$ vs. $33 \%$; $p=0.047)$. Headache was more common in those with confirmed or probable acute rickettsial infection than those with no acute rickettsial infection (77\% vs. $59 \%$; p $=0.004)$, especially among those with SFGR or TGR $(81 \%)$ compared with $O$. tsutsugamushi $(76 \%)$ or neither (59\%) (Appendix Tables 1-3). Dizziness was more frequent in those with confirmed and probable acute rickettsial infections (63\%) than those with no rickettsial infection $(48 \%$; $p=0.029)$; however, vomiting was less common (32\% vs. $46 \%$; $p=$ 0.035). Hearing loss was more common in those with acute $(9 \%)$ compared with no rickettsial infection $(2 \% ; p=0.023)$; this finding was most pronounced in confirmed O. tsutsugamushi infection (19\% [95\% 
CI 7\%-39\%]; $\mathrm{p}=0.004$ ) but also present in SFGR (18\% [95\% CI 4\%-43\%]; $p=0.021)$. Thirteen $(11 \%)$ patients with acute rickettsioses had a maculopapular rash, but none had an eschar.

Provisional clinical diagnoses among patients with confirmed acute rickettsioses included 7 $(14 \%)$ dengue, $6(12 \%)$ acute undifferentiated fever, $6(12 \%)$ community-acquired pneumonia, 5 $(10 \%)$ urinary tract infection, $4(8 \%)$ leptospirosis, $4(8 \%)$ gastroenteritis, and $23(47 \%)$ other diagnoses. No patient with or without confirmed rickettsial infection had a provisional diagnosis of acute rickettsial infection.

Anemia occurred in 24 (51\%) patients with acute rickettsioses and hematologic results available, and we noted a hemoglobin level of $<80 \mathrm{~g} / \mathrm{L}$ in 3 adults (Appendix Table 4). Peripheral leukocyte counts, renal function after controlling for age, and acute kidney injury, observed in $6 / 33$ patients $(18 \%)$, were similar in patients with and without acute rickettsioses.

Doxycycline was administered to $2(4 \%)$ patients with confirmed acute rickettsioses (both $O$. tsutsugamushi infections), albeit in both cases for a provisional diagnosis of gastroenteritis. None of the 6 rickettsial case-patients with a provisional diagnosis of acute undifferentiated fever were treated with doxycycline.

\section{Epidemiologic Features of Patients with Confirmed Acute or Past Rickettsial Infections}

Patients with acute or past rickettsial infections were older than those without (median age 43 vs. 24 years; $\mathrm{p}<0.001$ ); however, the distribution by sex was similar (57\% male among those with past rickettsial infection vs. 52\% among those without rickettsial infection). Most patients (67\%) reported rural residence (Appendix Table 5). Recently spending time in forest areas was more common (OR 2.1 [IQR $1.0-4.3] ; p=0.037)$ in patients with acute or past rickettsial infection (14\%) than patients with neither $(7 \%)$, including staying overnight in the forest for those with scrub typhus $(11 \% ; p=0.047)$. Patients were more likely to report a primary occupation as a rubber tapper (OR 5.9 [IQR 1.9-18.5]; $p=0.002$ ) for those with acute or past rickettsial infections (10\%) than for patients without rickettsioses $(2 \%)$. Of the 13 rubber tappers with seroprevalent rickettsioses, 3 (23\%) had confirmed acute scrub typhus and 5 (38\%) past scrub typhus. Farmers were also more likely (OR 2.8 [IQR 1.0-7.3]; $\mathrm{p}=0.041$ ) to have $(8 \%)$ than not to have (3\%) seroprevalent rickettsial infections. Patients who were unemployed had an increased risk of rickettsioses (OR 1.9 [IQR 1.0-3.5]; $\mathrm{p}=0.042$ ), of whom $4 / 24$ had a recent travel history. No other occupations were associated with an increased risk of seroprevalent rickettsioses.

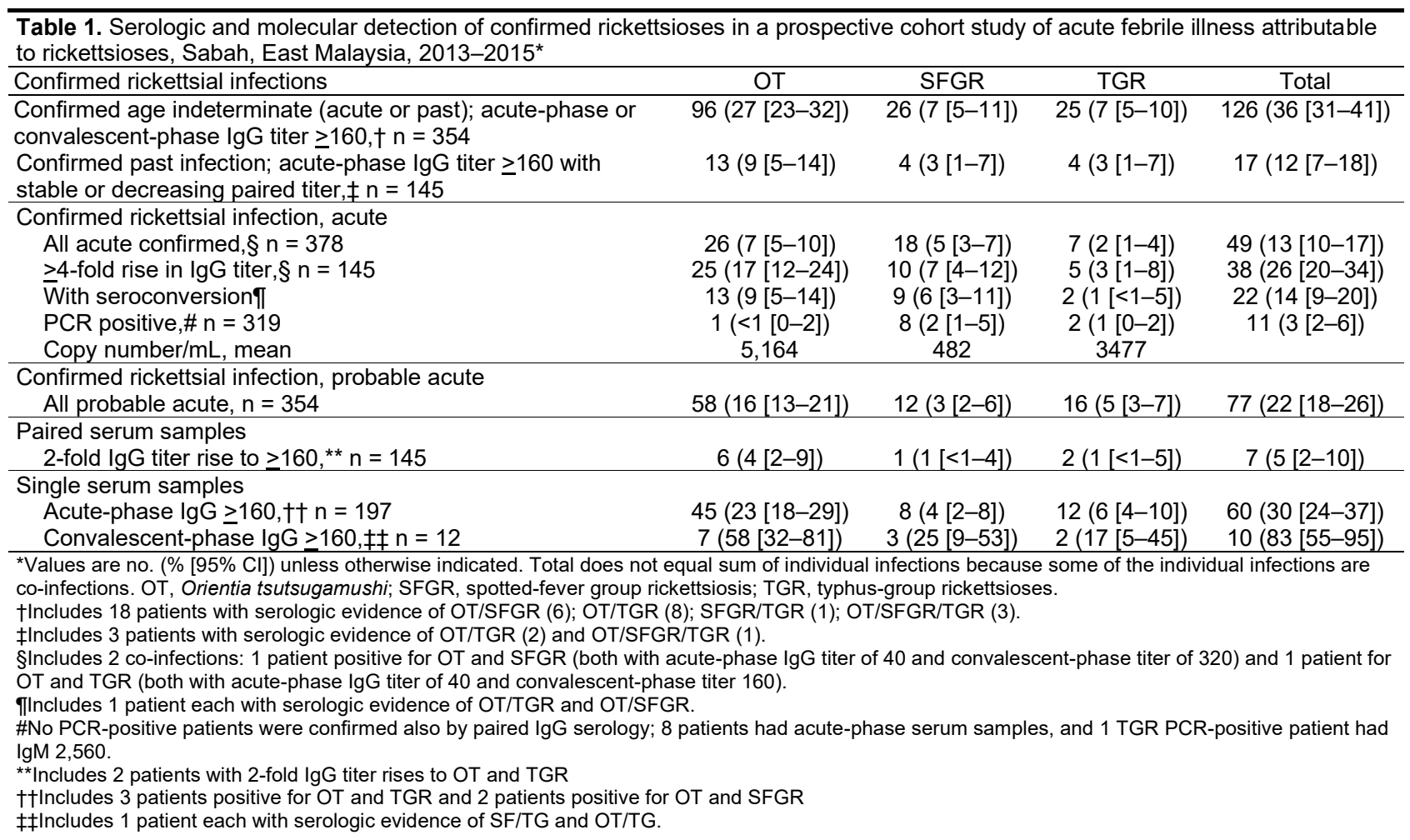




\section{Discussion}

Although rickettsial infections were first documented in Malaysia in 1925 (17), few cases are currently confirmed and reported nationally (18). Paired serum samples are infrequently obtained, and IFA requires expertise; both these factors limit diagnostic efforts. By confirming acute infection based on a 4-fold rise in IgG titer, PCR positivity, or both, we documented and characterized rickettsioses as an important cause of nonmalarial AFI in Malaysia (Figure 2). We found definitive serologic evidence of acute or past rickettsioses in $36 \%$ of patients and confirmed acute infections in $26 \%$ of patients. Scrub typhus (O. tsutsugamushi infection) was the most common rickettsiosis, especially among rural residents.
Acute-phase IgM demonstrated insufficient sensitivity for identifying acute rickettsioses. Moreover, acute-phase IgG, which cannot distinguish acute from past rickettsioses, is an imperfect measure of seroprevalence because antibodies decline over time even in patients who are not treated (19). PCR also detected few acute infections compared with paired serologic tests. Rickettsial infections were unsuspected clinical$1 y$, and nearly all were untreated. Therefore, increased clinical awareness, improved diagnostic tools, and empirical use of doxycycline are essential to reduce illness and death attributable to rickettsioses.

Confirmation of acute rickettsioses as a major cause of AFI in Sabah, Malaysia, is to be expected because rickettsioses have been identified elsewhere

\begin{tabular}{|c|c|c|c|c|c|c|}
\hline Characteristic & $\begin{array}{l}\text { Confirmed } \\
\text { acute } \\
\text { rickettsial } \\
\text { infection }\end{array}$ & $\begin{array}{l}\text { Probable } \\
\text { acute } \\
\text { rickettsial } \\
\text { infection }\end{array}$ & $\begin{array}{c}\text { p value for } \\
\text { confirmed vs. } \\
\text { probable acute } \\
\text { infection }\end{array}$ & $\begin{array}{c}\text { No } \\
\text { rickettsial } \\
\text { infection }\end{array}$ & $\begin{array}{l}\text { p value for } \\
\text { confirmed or } \\
\text { probable acute } \\
\text { vs. no infection }\end{array}$ & $\begin{array}{l}\mathrm{p} \text { value for } \\
\text { confirmed } \\
\text { acute vs. no } \\
\text { infection }\end{array}$ \\
\hline No. patients & 49 & 77 & NA & 102 & NA & NA \\
\hline \multicolumn{7}{|l|}{ Demographics } \\
\hline Age, median, y (IQR) & $39(2-56)$ & $43(28-62)$ & 0.32 & $29(8-55)$ & 0.007 & 0.15 \\
\hline Child $<15$ y of age & $8(16)$ & $3(4)$ & 0.023 & $29(28)$ & $<0.001$ & 0.22 \\
\hline \multicolumn{7}{|l|}{ Sex } \\
\hline $\mathrm{M}$ & $31(63)$ & $48(62)$ & 0.92 & $48(47)$ & 0.029 & 0.20 \\
\hline $\mathrm{F}$ & $18(37)$ & $29(38)$ & & $54(53)$ & & \\
\hline \multicolumn{7}{|l|}{ Symptoms } \\
\hline Symptoms data available & $48(98)$ & $75(97)$ & 0.99 & $102(100)$ & 0.99 & 0.99 \\
\hline Fever duration, median days (IQR) & $2(2-4)$ & $3(2-5)$ & 0.46 & $3(1-4)$ & 0.64 & 0.89 \\
\hline Headache & $36(75)$ & $60(80)$ & 0.65 & $60(59)$ & 0.004 & 0.05 \\
\hline Dizziness & $33(69)$ & $44(59)$ & 0.23 & $49(48)$ & 0.029 & 0.017 \\
\hline Confusion & $5(10)$ & $5(7)$ & 0.34 & $9(9)$ & 0.75 & 0.75 \\
\hline Vision changes & $8(17)$ & $10(13)$ & 0.56 & $11(11)$ & 0.42 & 0.31 \\
\hline Retro-orbital pain & $11(23)$ & $19(25)$ & 0.99 & $22(22)$ & 0.82 & 0.85 \\
\hline Hearing loss & $7(15)$ & $4(5)$ & 0.11 & $2(2)$ & 0.023 & 0.005 \\
\hline Coryza & $16(33)$ & $22(30)$ & 0.50 & $32(31)$ & 0.82 & 0.81 \\
\hline Cough & $23(48)$ & $34(45)$ & 0.59 & $41(40)$ & 0.48 & 0.37 \\
\hline Dyspnea & $16(33)$ & 13 (17) & 0.047 & $27(26)$ & 0.64 & 0.39 \\
\hline Joint pain & $20(42)$ & $29(39)$ & 0.62 & $36(35)$ & 0.57 & 0.45 \\
\hline Muscle pain & $14(29)$ & 13 (17) & 0.08 & $23(23)$ & 0.81 & 0.38 \\
\hline Lethargy & $32(67)$ & $45(60)$ & 0.37 & $59(58)$ & 0.54 & 0.30 \\
\hline Nausea & $16(33)$ & $34(46)$ & 0.16 & $44(44)$ & 0.71 & 0.23 \\
\hline Vomiting & $13(27)$ & $27(36)$ & 0.32 & 47 (46) & 0.035 & 0.027 \\
\hline Abdominal pain & $20(42)$ & $33(44)$ & 0.56 & $41(40)$ & 0.48 & 0.86 \\
\hline Loss of appetite & $34(71)$ & 49 (66) & 0.43 & $70(68)$ & 0.76 & 0.79 \\
\hline Diarrhea & $12(25)$ & $20(27)$ & 0.67 & $32(31)$ & 0.49 & 0.42 \\
\hline Dysuria & $4(8)$ & $6(8)$ & 0.99 & $6(6)$ & 0.61 & 0.46 \\
\hline \multicolumn{7}{|l|}{ Signs } \\
\hline Conjunctival suffusion & $0 / 46(0)$ & $0 / 73(0)$ & NA & 2/95 (2) & 0.21 & 0.99 \\
\hline Respiratory distress $†$ & $10(21)$ & $21(27)$ & 0.67 & $34(33)$ & 0.21 & 0.17 \\
\hline Respiratory crepitations on auscultation & $5(10)$ & $12 / 74(16)$ & 0.25 & 18 (18) & 0.32 & 0.33 \\
\hline Abnormal chest radiograph result & $8 / 14(57)$ & $18 / 31(58)$ & 0.50 & 23/39 (59) & 0.70 & 0.99 \\
\hline Maculopapular rash & $4(8)$ & 10/75 (13) & 0.37 & $6(6)$ & 0.23 & 0.73 \\
\hline Eschar & $0 / 47(0)$ & 0/74 (0) & NA & $0 / 101(0)$ & NA & NA \\
\hline Lymphadenopathy & 0/46 (0) & 2/72 (3) & 0.53 & 3/98 (3) & 0.67 & 0.55 \\
\hline Abdominal tenderness & $7 / 44(16)$ & $5 / 49(10)$ & 0.36 & $11 / 91$ (12) & 0.76 & 0.59 \\
\hline Hepatomegaly & $10(21)$ & $9 / 75(12)$ & 0.13 & 10 (10) & 0.17 & 0.06 \\
\hline Splenomegaly & $3 / 41(7)$ & $2 / 73(3)$ & 0.33 & $2 / 89(2)$ & 0.46 & 0.33 \\
\hline
\end{tabular}

*Values are no. (\%) unless indicated. Bold indicates a statistically significant difference $(p<0.05)$. Results are from time of study enrolment unless indicated. IQR, interquartile range; NA, not applicable.

†Respiratory rate $\geq 30$ breaths/min or tissue oxygen saturation $<96 \%$ on room air. 


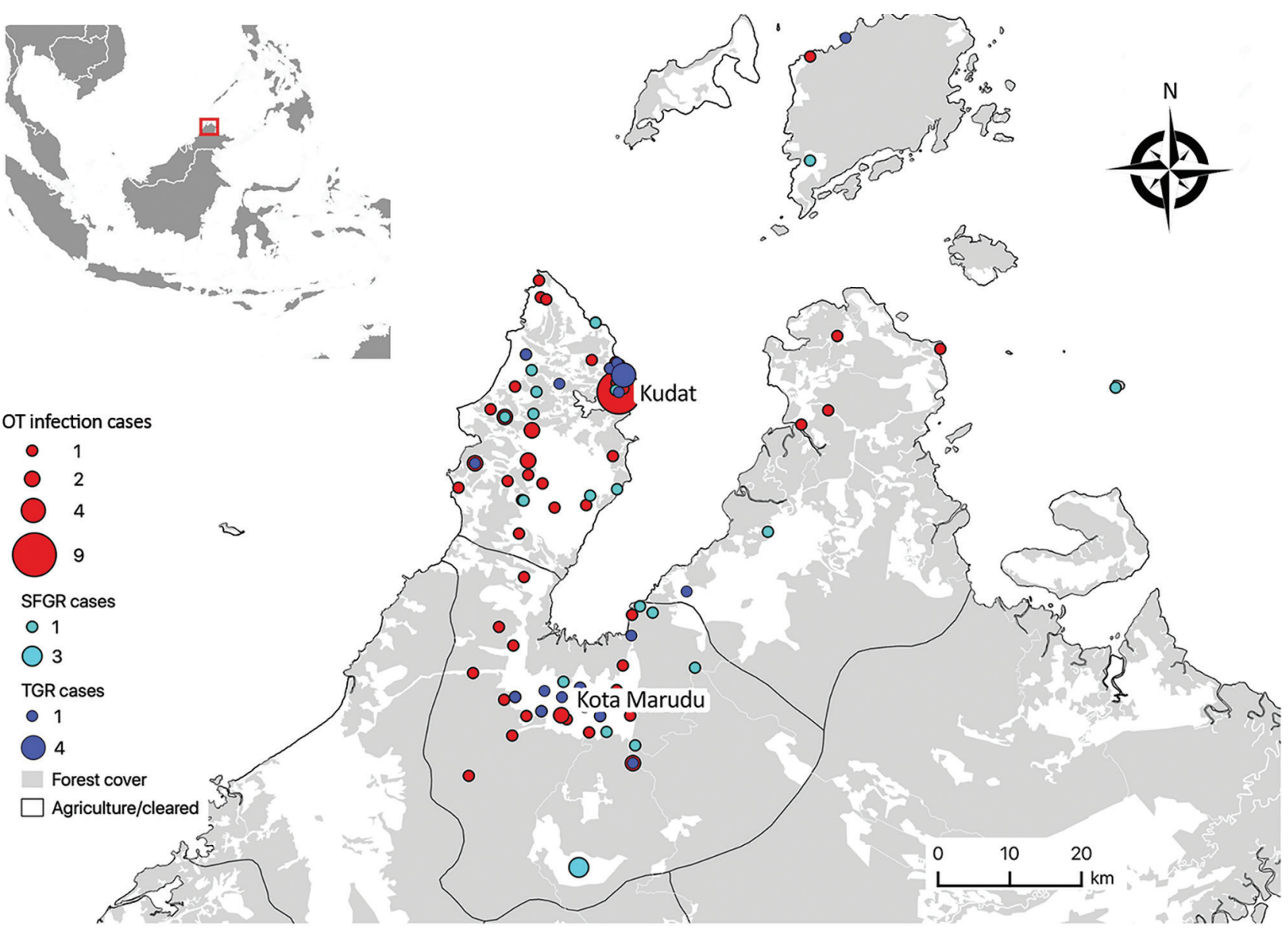

Figure 2. Village-level geographic distribution of confirmed acute and probable acute rickettsioses cases in a prospective cohort study of acute febrile illness attributable to rickettsioses, Sabah, East Malaysia, 2013-2015. Inset map shows study area in Sabah, Malaysia. OT, Orientia tsutsugamushi infection; SFGR, spotted-fever group rickettsiosis; TGR, typhus-group rickettsioses.

in the region. A large multicenter study in Thailand, Vietnam, and Indonesia showed that $6 \%$ of 1,578 enrolled patients had serologically confirmed acute rickettsioses (20). Other rigorous clinical and crosssectional studies have demonstrated marked spatial heterogeneity in seroprevalence of rickettsioses (21), including $1 \%$ in Thailand (22), 33\% in areas bordering Myanmar (23), $7 \%$ to $27 \%$ in Laos (24-26), and up to $10 \%$ in parts of Indonesia $(27,28)$. Our study suggests that rickettsioses are at least as common $(26 \%)$ in Malaysia as elsewhere in the region.

Rickettsioses are likely to be reemerging in East Malaysia. Despite the presence of a common vector of scrub typhus, Leptotrombidium (L.) deliense mites, being documented in coastal areas for decades (29), a single statewide study (including Kudat District) in the 1980s reported no acute rickettsioses among 383 febrile patients with paired serum samples tested by IFA (30). Sero-epidemiologic findings from 837 persons at that time demonstrated a low seroprevalence of $0.8 \%$ for O. tsutsugamushi, $8.6 \%$ for SFGR, and $0 \%$ for TGR (30). At the Sabah state tertiary referral hospital during 1994-1999, a total of 11,037 samples from febrile patients sent for acute IgG or IgM testing showed a comparatively low seroprevalence of $2.6 \%$ for O. tsutsugamushi, $1.3 \%$ for TGR, and $1.6 \%$ for SFGR (5). However, the inability to confirm acute rickettsioses, even retrospectively, if paired serum samples are not obtained has constrained broader public health understanding of their epidemiology and disease prevalence among the at-risk population in Sabah.

The epidemiology of rickettsial infections in this study is consistent with previous reports of scrub typhus and spotted fever rickettsioses described in rural areas in Southeast Asia (31); those reports indicated that infections were more commonly found in male adults, in agricultural, plantation, or forestry workers $(8,28,32,33)$, and in persons with lower socio-economic status $(21,34)$. The presence of typhus group rickettsioses in rural residents in our study 
population, despite generally being reported more frequently in urban populations (31), highlights the need for further studies to better understand the epidemiology of rickettsioses in Malaysia and to design appropriate control measures. Because SFGR and TGR cross-reactivity by IFA is expected, testing for all 3 rickettsioses (O. tsutsugamushi infection, SFGR, and TGR) is important.

Factors explaining a potential increase in rickettsioses in Sabah could in part be related to changes in human land use (10) and the relationship with occupational or travel behavior and vector bionomics (35). The effect of habitat on O. tsutsugamushi seroprevalence has previously been evaluated in peninsular Malaysia; higher seropositivity overall was observed in indigenous adults with exposure to intact forest areas $(73 \%)$ compared with forest fringe (48\%) and village areas (8\%) (4). These findings are broadly consistent with the association in our study between persons with acute or past rickettsioses and a history of extended forest exposure. Rubber plantation workers in peninsular Malaysia have been reported to have higher SFGR prevalence $(40 \%)$, compared with O. tsutsugamushi (8\%) and TGR (1\%) (36). In contrast, although those identified as rubber tappers in our study had high seroprevalence to rickettsioses overall, most of these infections were attributable to O. tsutsugamushi.

We found that patients with acute rickettsioses had clinical findings similar to those without acute rickettsioses, with the exception of more frequent dizziness and hearing loss and less frequent vomiting. Detection of hearing loss might assist in clinical recognition of rickettsioses and has been reported in up to one third of patients with acute scrub typhus (37) as well as in those with SFGR (38). Eschar occurs most frequently on the anterior chest in women and the groin in men with scrub typhus (39); the relative rarity of eschar and higher frequency of hepatomegaly found in our study was also previously described in Malaysia (2). We observed severe cases of acute kidney injury in a small number of patients; however, acute kidney injury remains clinically important because of the subsequent increased risk for chronic kidney disease (40).

Common laboratory markers, such as abnormalities in leukocyte count, thrombocytopenia, anemia, and raised liver transaminases, were all nonspecific (31). Neutropenia occurred in 1 patient with acute scrub typhus, despite this sign being reported as a potentially useful clinical tool to differentiate scrub typhus from dengue (41). AFI was primarily attributed to dengue by treating clinicians, who initiated dengue fluid management protocols. In other cases, empiric treatment of suspected typhoid or leptospirosis was initiated with cephalosporins or penicillins, which are ineffective against rickettsioses. Treatment with doxycycline is recommended by the Malaysia Ministry of Health (42) because an estimated $6 \%$ of untreated scrub typhus cases in Asia are fatal (43); however, we found that rickettsioses were unsuspected and untreated.

Tools to support early diagnosis and local evidence-based clinical algorithms to guide directed and empirical treatment of undifferentiated AFI are needed. Diagnosis of acute rickettsioses remains problematic. Orientia and Rickettsia spp. are obligate intracellular bacteria predominantly targeting endothelial cells $(44,45)$; hence, real-time PCR on peripheral blood is insensitive for the detection of acute rickettsioses $(15,19,46)$. Incomplete follow-up meant the sensitivity of PCR could not be directly measured against gold-standard IFA serologic diagnosis for SFGR and TGR, although low PCR sensitivity of $18 \%$ has been reported (19). Longstanding technical difficulties with IFA also include the need for extensively trained readers and expensive equipment. Conventional use of antibody detection against only 3 major O. tsutsugamushi antigen groups and serologic cross-reactivity further limits accurate diagnoses; however, high antigenic variation has been observed across Southeast Asia $(47,48)$. We found that acute-phase IgM was a poor predictor of acute rickettsial infection even in the setting of acute scrub typhus, underscoring the importance of increased clinical awareness, improved diagnostic tools, and empiric treatment of those with epidemiologic or clinical risk factors.

The main limitation of this study was the relatively low proportion (41\%) of patients with both acute-phase and convalescent-phase serum samples compared with our previous studies $(17,18)$. For this reason, we also performed PCR and tested for IgM, even though sensitivity of the former and specificity of the latter are limited for identifying acute rickettsioses (19). Our estimates of the seroprevalence of rickettsioses and incidence of confirmed acute rickettsioses are conservative. First, only half of patients returned for convalescent follow-up, yet most confirmed acute rickettsioses were seroconversions rather than based on 4-fold rises in titer alone. Second, over half of patients who did have a convalescent-phase serum sample obtained had it obtained earlier than the recommended $\geq 14$ days. In experimental human SFGR infection, $\geq 3$ weeks were required before the geometric mean titer of infected volunteers reached 
diagnostic IgG titers (49). Third, antibody detection of different antigen groups such as that observed in O. tsutsugamushi was based on cross-reactivity to the single Karp strain. Finally, patients with seroconversions indicative of past infection might be missed by a screening strategy that entails testing convalescentphase serum samples first.

To better estimate the actual prevalence of acute rickettsioses, we additionally delineated patients with confirmed rickettsioses with likely acute infection (patients with rising or single IgG titers of $\geq 160$ ) as having probable acute infections. The likely underestimation of acute infections caused by diagnostic uncertainty is supported by the similarity in initial clinical and laboratory findings between confirmed and probable acute rickettsioses. The burden and clinical features of rickettsioses are likely underestimated in our study not only because of underreporting of laboratory-confirmed cases but also because persons with mild illness, nonspecific features, and limited physical or economic ability to travel to hospital and persons living in remote areas might not have sought care. Similarly, persons with severe disease might have sought care at or been transferred to a tertiary hospital before study enrollment or died before care they sought or obtained care. TGR prevalence is also potentially underestimated because our population was mainly rural rather than urban. However, our data are likely generalizable to similar populations in Sabah and potentially neighboring areas.

In conclusion, rickettsioses are prevalent among persons with nonmalarial AFI in Sabah, Malaysia. A comprehensive prospective study of rickettsial infections across Malaysia is warranted to define the totality of regional risk. Improved diagnostic tools are urgently needed; in their absence, increased clinical suspicion and empirical doxycycline might avert disease and death attributable to rickettsioses.

\section{Acknowledgments}

We thank the participants in this study and the clinical and laboratory research staff, including Emma Istiana binti Israk, Sitti Saimah binti Sakam, Norfarain binti Mohamed Yassin, Salwah Hamit binti Hamid, Noorazela binti Mohamed Yassin, Areys Benjamin, Wilhelmina Nevir, Siti Norfazaziwantie binti Redzuan, and Maslianah binti Sintom. We express sincere appreciation also to the hospital directors at the study sites. We thank the Director-General of Health, Ministry of Health, Malaysia, for permission to publish this study. We also thank Emily Clemens and Amanda Olivier for excellent technical support with serologic and molecular testing.
This study was supported by the Malaysia Ministry of Health (grant no. 2014-26), the Australian National Health and Medical Research Council (program no. 1037304; fellowship no. 605831 to T.W.Y.; scholarship no. 1074795 and fellowship no. 1138860 to M.J.G.; fellowship no. 1135820 to N.M.A.), the US National Institutes of Allergy and Infectious Diseases (grant no. R03 AI111300 to M.E.R.), and the Uniformed Services University for the Health Sciences (grant no. PAT-74-3977 to J.S.D.).

\section{About the Author}

Dr. Grigg is a senior research fellow at Menzies School of Health Research and Charles Darwin University, Australia. His primary research interests include the epidemiology, diagnosis and treatment of Plasmodium knowlesi and other Plasmodium species infections causing malaria in Malaysia and Indonesia; other tropical and zoonotic/vectorborne infectious diseases; and the health of Indigenous peoples in remote Northern Territory communities in Australia.

\section{References}

1. McQuiston JH, Wiedeman C, Singleton J, Carpenter LR, McElroy K, Mosites E, et al. Inadequacy of IgM antibody tests for diagnosis of Rocky Mountain Spotted Fever. Am J Trop Med Hyg. 2014;91:767-70. https:// doi.org/10.4269/ ajtmh.14-0123

2. Brown GW, Robinson DM, Huxsoll DL, Ng TS, Lim KJ, Sannasey G. Scrub typhus: a common cause of illness in indigenous populations. Trans R Soc Trop Med Hyg. 1976;70:444-8. https:// doi.org/10.1016/0035-9203(76) 90127-9

3. Brown GW, Shirai A, Jegathesan M, Burke DS, Twartz JC, Saunders JP, et al. Febrile illness in Malaysia - an analysis of 1,629 hospitalized patients. Am J Trop Med Hyg. 1984;33:311-5. https:// doi.org/10.4269/ajtmh.1984.33.311

4. Cadigan FC Jr, Andre RG, Bolton M, Gan E, Walker JS. The effect of habitat on the prevalence of human scrub typhus in Malaysia. Trans R Soc Trop Med Hyg. 1972;66:582-7. https:/ / doi.org/10.1016/0035-9203(72)90303-3

5. Tay ST, Rohani MY. The use of the indirect immunoperoxidase test for the serodiagnosis of rickettsial diseases in Malaysia. Southeast Asian J Trop Med Public Health. 2002;33:314-20.

6. Ministry of Health Malaysia. Case definitions for infectious diseases in Malaysia. 3rd edition. 2017 Jan 1 [cited 2019 Jan 12]. http:/ / www.moh.gov.my/index.php

7. Ministry of Health Malaysia. Health indicators (Pentunjuk Kesihatan), 2015 [cited 2019 Jan 12] http://www.moh.gov.my/images/gallery/publications/ Petunjuk\%20Kesihatan\%202016.pdf

8. Tay ST, Ho TM, Rohani MY, Devi S. Antibodies to Orientia tsutsugamushi, Rickettsia typhi and spotted fever group rickettsiae among febrile patients in rural areas of Malaysia. Trans R Soc Trop Med Hyg. 2000;94:280-4. https://doi.org/10.1016/S0035-9203(00)90322-5

9. Malaysia Government Department of Statistics. Population and housing census, Malaysia. 2010 [cited 2018 Sep 15]. https:/ / www.statistics.gov.my/mycensus2010/ images/stories/files/Laporan_Kiraan_Permulaan2010.pdf 
10. Bryan JE, Shearman PL, Asner GP, Knapp DE, Aoro G, Lokes B. Extreme differences in forest degradation in Borneo: comparing practices in Sarawak, Sabah, and Brunei. PLoS One. 2013;8(7):e69679.

11. World Health Organization. Haemoglobin concentrations for the diagnosis of anaemia and assessment of severity. 2011 [cited 2018 Oct 10]. http://www.who.int/vmnis/indicators/ haemoglobin.pdf

12. KDIGO clinical practice guidelines for acute kidney injury. Kidney Int Suppl. 2012;2:1-138.

13. Reller ME, Chikeka I, Miles JJ, Dumler JS, Woods CW, Mayorga $\mathrm{O}$, et al. First identification and description of rickettsioses and $\mathrm{Q}$ fever as causes of acute febrile illness in Nicaragua. PLoS Negl Trop Dis. 2016;10:e0005185. https://doi.org/10.1371/journal.pntd.0005185

14. Reller ME, Bodinayake C, Nagahawatte A, Devasiri V, Kodikara-Arachichi W, Strouse JJ, et al. Unsuspected rickettsioses among patients with acute febrile illness, Sri Lanka, 2007. Emerg Infect Dis. 2012;18:825-9. https://doi.org/10.3201/eid1805.111563

15. Prakash JAJ, Reller ME, Barat N, Dumler JS. Assessment of a quantitative multiplex $5^{\prime}$ nuclease real-time PCR for spotted fever and typhus group rickettsioses and Orientia tsutsugamushi. Clin Microbiol Infect. 2009;15(Suppl 2):292-3. https://doi.org/10.1111/j.1469-0691.2008.02242.x

16. US Centers for Disease Control and Prevention. Spotted fever rickettsiosis (Rickettsia spp.) 2010 case definition. 2010 [cited 2018 Oct 5]. https:// wwwn.cdc.gov/nndss/ conditions/spotted-fever-rickettsiosis/case-definition/2010

17. Fletcher W, Lesslar JE. Tropical typhus in the Federated Malay States with a compilation on epidemic typhus. Bull Inst Med Res. 1925;2:88.

18. Malaysia Ministry of Health. Health indicators (Pentunjuk Kesihatan) 2016 [cited 2018 Nov 23]. http:/ / www.moh.gov. my/images/gallery/publications/Petunjuk\%20Kesihatan \%202017.pdf

19. Paris DH, Dumler JS. State of the art of diagnosis of rickettsial diseases: the use of blood specimens for diagnosis of scrub typhus, spotted fever group rickettsiosis, and murine typhus. Curr Opin Infect Dis. 2016;29:433-9. https://doi.org/10.1097/QCO.0000000000000298

20. Southeast Asia Infectious Disease Clinical Research Network. Causes and outcomes of sepsis in southeast Asia: a multinational multicentre cross-sectional study. Lancet Glob Health. 2017;5:e157-67. https:/ / doi.org/ 10.1016/S2214-109X(17)30007-4

21. Vallée J, Thaojaikong T, Moore CE, Phetsouvanh R, Richards AL, Souris M, et al. Contrasting spatial distribution and risk factors for past infection with scrub typhus and murine typhus in Vientiane City, Lao PDR. PLoS Negl Trop Dis. 2010;4:e909. https:// doi.org/10.1371/ journal.pntd.0000909

22. Pradutkanchana J, Pradutkanchana S, Kemapanmanus M, Wuthipum N, Silpapojakul K. The etiology of acute pyrexia of unknown origin in children after a flood. Southeast Asian J Trop Med Public Health. 2003;34:175-8.

23. Parola P, Miller RS, McDaniel P, Telford SR III, Rolain J-M, Wongsrichanalai $\mathrm{C}$, et al. Emerging rickettsioses of the Thai-Myanmar border. Emerg Infect Dis. 2003;9:592-5. https://doi.org/10.3201/eid0905.020511

24. Syhavong B, Rasachack B, Smythe L, Rolain J-M, Roque-Afonso A-M, Jenjaroen $\mathrm{K}$, et al. The infective causes of hepatitis and jaundice amongst hospitalised patients in Vientiane, Laos. Trans R Soc Trop Med Hyg. 2010;104:475-83. https://doi.org/10.1016/ j.trstmh.2010.03.002
25. Phongmany S, Rolain J-M, Phetsouvanh R, Blacksell SD, Soukkhaseum V, Rasachack B, et al. Rickettsial infections and fever, Vientiane, Laos. Emerg Infect Dis. 2006;12:256-62. https://doi.org/10.3201/eid1202.050900

26. Mayxay M, Castonguay-Vanier J, Chansamouth V, Dubot-Pérès A, Paris DHP, Phetsouvanh R, et al. Causes of non-malarial fever in Laos: a prospective study. Lancet Glob Health. 2013;1:e46-54. https:// doi.org/10.1016/ S2214-109X(13)70008-1

27. Gasem MH, Wagenaar JFP, Goris MGA, Adi MS, Isbandrio BB, Hartskeerl RA, et al. Murine typhus and leptospirosis as causes of acute undifferentiated fever, Indonesia. Emerg Infect Dis. 2009;15:975-7. https:/ / doi.org/ 10.3201/eid1506.081405

28. Richards AL, Ratiwayanto S, Rahardjo E, Kelly DJ, Dasch GA, Fryauff DJ, et al. Serologic evidence of infection with ehrlichiae and spotted fever group rickettsiae among residents of Gag Island, Indonesia. Am J Trop Med Hyg. 2003;68:480-4. https://doi.org/10.4269/ajtmh.2003.68.480

29. Dohany AL, Phang OW, Rapmund G. Chigger (Acarina: Trombiculidae) surveys of the west coast beaches of Sabah and Sarawak. Southeast Asian J Trop Med Public Health. 1977;8:200-6.

30. Taylor AC, Hii J, Kelly DJ, Davis DR, Lewis GE Jr. A serological survey of scrub, tick, and endemic typhus in Sabah, East Malaysia. Southeast Asian J Trop Med Public Health. 1986;17:613-9.

31. Aung AK, Spelman DW, Murray RJ, Graves S. Rickettsial infections in Southeast Asia: implications for local populace and febrile returned travelers. Am J Trop Med Hyg. 2014;91:451-60. https:// doi.org/10.4269/ajtmh.14-0191

32. Suttinont $\mathrm{C}$, Losuwanaluk K, Niwatayakul K, Hoontrakul S, Intaranongpai W, Silpasakorn S, et al. Causes of acute, undifferentiated, febrile illness in rural Thailand: results of a prospective observational study. Ann Trop Med Parasitol. 2006;100:363-70. https:// doi.org/10.1179/136485906X112158

33. Suputtamongkol Y, Suttinont C, Niwatayakul K, Hoontrakul S, Limpaiboon R, Chierakul W, et al. Epidemiology and clinical aspects of rickettsioses in Thailand. Ann N Y Acad Sci. 2009;1166:172-9. https://doi.org/10.1111/j.1749-6632.2009.04514.x

34. Tay ST, Mohamed Zan HA, Lim YAL, Ngui R. Antibody prevalence and factors associated with exposure to Orientia tsutsugamushi in different aboriginal subgroups in West Malaysia. PLoS Negl Trop Dis. 2013;7:e2341.

35. Kilpatrick AM, Randolph SE. Drivers, dynamics, and control of emerging vector-borne zoonotic diseases. Lancet. 2012;380:1946-55. https:/ / doi.org/10.1016/ S0140-6736(12)61151-9

36. Tee TS, Kamalanathan M, Suan KA, Chun SS, Ming HT, Yasin RM, et al. Seroepidemiologic survey of Orientia tsutsugamushi, Rickettsia typhi, and TT118 spotted fever group rickettsiae in rubber estate workers in Malaysia. Am J Trop Med Hyg. 1999;61:73-7. https:/ / doi.org/10.4269/ ajtmh.1999.61.73

37. Noad KB, Haymaker W. The neurological features of Tsutsugamushi fever, with special reference to deafness. Brain. 1953;76:113-31. https:/ / doi.org/10.1093/brain/ 76.1 .113

38. Sexton DJ. Acute hearing loss and rickettsial diseases. Clin Infect Dis. 2006;42:1506. https://doi.org/10.1086/ 503682

39. Kim D-M, Won KJ, Park CY, Yu KD, Kim HS, Yang TY, et al. Distribution of eschars on the body of scrub typhus patients: a prospective study. Am J Trop Med Hyg. 2007;76:806-9. https://doi.org/10.4269/ajtmh.2007.76.806 
40. Hsu RK, Hsu C-Y. The role of acute kidney injury in chronic kidney disease. Semin Nephrol. 2016;36:283-92. https:/ / doi.org/10.1016/j.semnephrol.2016.05.005

41. Raby E, Dyer JR. Endemic (murine) typhus in returned travelers from Asia, a case series: clues to early diagnosis and comparison with dengue. Am J Trop Med Hyg. 2013;88:701-3. https://doi.org/10.4269/ajtmh.12-0590

42. Ministry of Health Malaysia. National Antimicrobial Guideline. 2019 [cited 2019 Feb 12]. https:/ / www.pharmacy. gov.my/v2/sites/default/files/document-upload/ national-antimicrobial-guideline-2019-full-version-3rdedition.pdf

43. Taylor AJ, Paris DH, Newton PN. A systematic review of mortality from untreated scrub typhus (Orientia tsutsugamushi). PLoS Negl Trop Dis. 2015;9:e0003971.

44. Valbuena G, Walker DH. Infection of the endothelium by members of the order Rickettsiales. Thromb Haemost. 2009;102:1071-9. https:// doi.org/10.1160/TH09-03-0186

45. Moron CG, Popov VL, Feng HM, Wear D, Walker DH. Identification of the target cells of Orientia tsutsugamushi in human cases of scrub typhus. Mod Pathol. 2001;14:752-9. https:// doi.org/10.1038/modpathol.3880385

46. Paris DH, Blacksell SD, Stenos J, Graves SR, Unsworth NB, Phetsouvanh R, et al. Real-time multiplex PCR assay for detection and differentiation of rickettsiae and orientiae. Trans
R Soc Trop Med Hyg. 2008;102:186-93. https:// doi.org/ 10.1016/j.trstmh.2007.11.001

47. Kelly DJ, Fuerst PA, Ching W-M, Richards AL. Scrub typhus: the geographic distribution of phenotypic and genotypic variants of Orientia tsutsugamushi. Clin Infect Dis. 2009;48 Suppl 3:S203-30.

48. Tay ST, Rohani MY, Ho TM, Devi S. Antigenic types of Orientia tsutsugamushi in Malaysia. Southeast Asian J Trop Med Public Health. 2002;33:557-64.

49. Clements ML, Dumler JS, Fiset P, Wisseman CL Jr, Snyder MJ, Levine MM. Serodiagnosis of Rocky Mountain spotted fever: comparison of IgM and IgG enzyme-linked immunosorbent assays and indirect fluorescent antibody test. J Infect Dis. 1983;148:876-80. https://doi.org/10.1093/infdis/148.5.876

Address for correspondence: Matthew Grigg, Global and Tropical Health Division, Menzies School of Health Research, PO Box 41096, Casuarina, Darwin 0811, Northern Territory, Australia; email: matthew.grigg@menzies.edu.au; Megan E. Reller, Division of Infectious Diseases and International Health, Department of Medicine, Duke University School of Medicine, 315 Trent Dr, Hanes House, Rm 250, Durham, NC 27710, USA; email: megan.reller@duke.edu

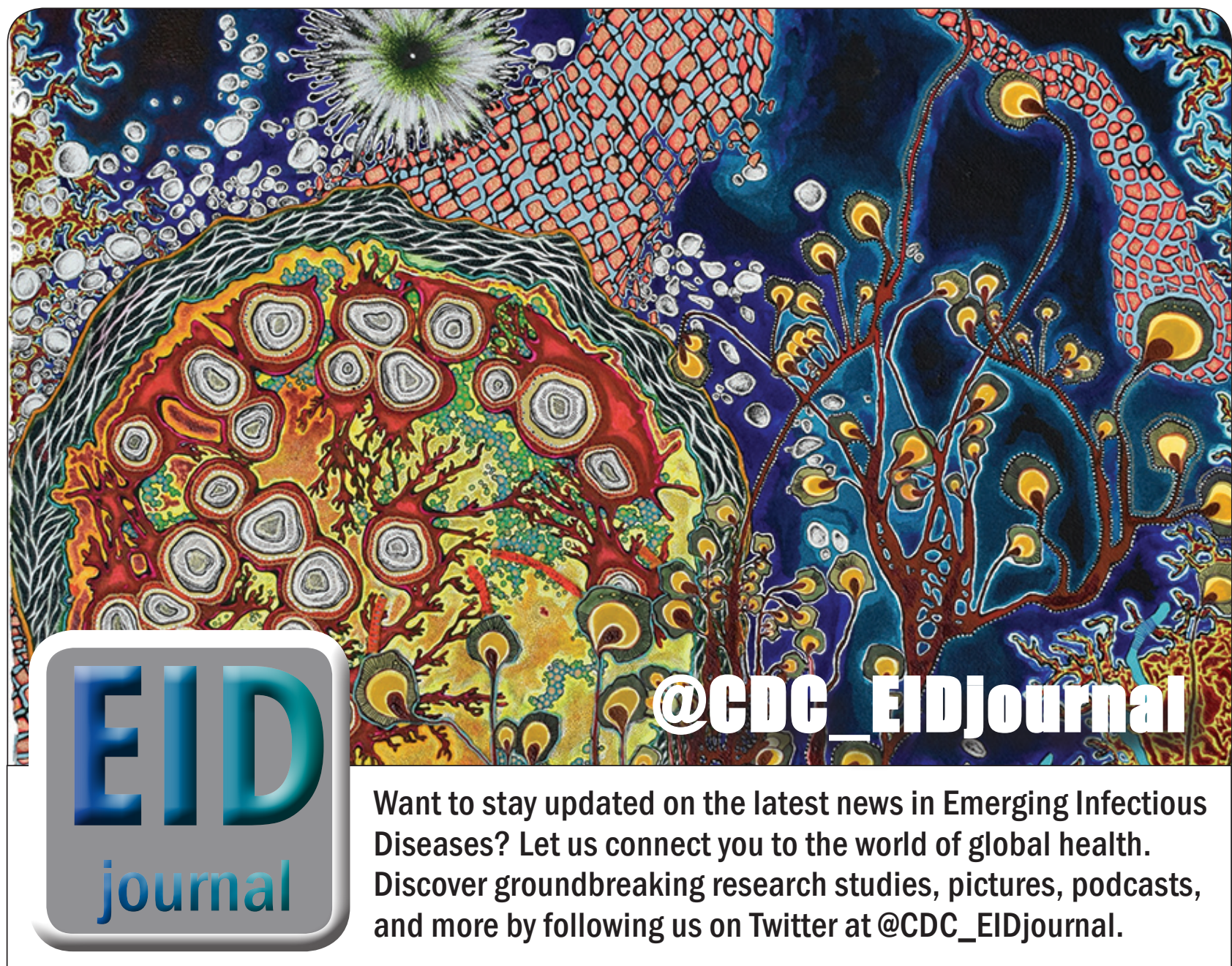

